\title{
Arquitetura para Modelagem de Estudante com Recursos de Interação por meio de ChatBots
}

\author{
Tiago Silveira Cherchiglia $^{1}$, Vinícius Alves Silva ${ }^{1}$, Hiran Nonato M. Ferreira ${ }^{1}$ \\ ${ }^{1}$ Instituto Federal de Educação, Ciência e Tecnologia do Sul de Minas Gerais \\ (IFSULDEMINAS) - Passos/MG, Brasil
}

\begin{abstract}
With the advancement in Artificial Intelligence techniques applied to the analysis and interpretation of human behavior, it is, increasingly easier, to apply such resources to improve the performance of people's daily tasks. When used in an educational environment, these techniques have improved the teaching/learning process. In this sense, this work proposes the definition of an architecture for integrating Chatbots into Educational Environments. In addition to improving the interaction process between environment, teachers and students, the proposed architecture aims to integrate student modeling resources in this communicational process.
\end{abstract}

Resumo. Com o avanço nas técnicas de Inteligência Artificial aplicadas à análise e interpretação do comportamento humano, está, cada vez mais simples, aplicar tais recursos para melhorar a realização de tarefas do cotidiano das pessoas. Quando empregadas a ambiente educacionais, essas técnicas têm trazido melhoria do processo ensino/aprendizado. Neste sentido, este trabalho propõe a definição de uma arquitetura para integração de Chatbots a Ambientes Educacionais. Além de melhorar o processo de interação entre ambiente, professores e estudantes, a arquitetura proposta busca integrar recursos de modelagem de estudante neste processo comunicacional.

\section{Introdução}

A utilização da tecnologia como mecanismo para auxiliar o processo de ensino/aprendizagem está cada vez mais presente no cotidiano das pessoas. Essas tecnologias baseiam-se em diversas técnicas da computação a fim de propor estratégias e abstrações para tornar o ensino mais simples, dinâmico e atrativo aos estudantes. Diversas abordagens são propostas com o intuito de auxiliar na personalização, na integração e na visualização de novas estratégias pedagógicas para apoiar atividades educacionais.

Um dos grandes desafios que são tratados atualmente no contexto dos Ambientes Virtuais de Aprendizagem (AVAs) diz respeito ao acompanhamento das capacidades cognitivas dos estudantes [Greiff et al. 2016, Nguyen et al. 2017]. Segundo Brusilovsky [Brusilovsky 2001], o processo de ensino é mais eficiente se for possível identificar o real estado de conhecimento de cada estudante individualmente, dessa forma, seria possível aos instrutores buscarem estratégias pedagógicas adequadas para sanar as limitações individualizadas de cada estudante.

Buscando auxiliar estudantes no processo de identificação das suas capacidades e limitações, Modelos Abertos de Estudantes (MAEs) têm sido propostos como ferramentas para "externalizar" informações manipuladas pelos Modelos de Estudantes (MEs) 
Tradicionais [Ferreira et al. 2017]. Esses modelos permitem que as informações que antes eram tratadas somente internamente pelo sistema, agora possam ser disponibilizadas para os estudantes. Com isso, os próprios estudantes conseguem perceber o seu estado de conhecimento e auto guiar suas estratégias de estudo.

É imprescindível que a comunicação entre sistema e estudante deva ser a mais eficiente possível. Sendo assim, para prover tal interação, esta pesquisa fez o uso de conceitos definidos pelos chatbots, robôs que se baseiam em Inteligência Artificial (IA) para permitir uma comunicação direta e efetiva com usuários humanos. Essa inteligência é obtida através de uma série de algoritmos que são capazes de "aprender" com base em uma série de dados previamente informados.

Com base nisso, esta pesquisa propõe uma abordagem para construção de MEs com capacidades para fornecer uma interação mais efetiva entre professores e estudantes. A abordagem proposta utiliza um sistema de chatbot como mecanismo para troca de mensagens, permitindo, com isso, a construção de um modelo inteligente, capaz de processar recursos linguísticos e interagir com estudantes buscando uma melhor efetividade do processo de ensino/aprendizagem.

\section{Background}

A informática vem se mostrando cada vez mais presente na educação. Principalmente através dos Sistemas Adaptativos e Inteligentes para Educação (SAIEs) que em definição são sistemas capazes de adaptar suas características para atender às necessidades dos estudantes, analisando características e informações importantes ajudando no melhoramento da aprendizagem [Durlach and Lesgold 2012].

SAIEs também podem ser utilizados em conjunto com chatbots para aprimorar e aumentar a eficácia da aprendizagem. Experiências iniciais como as descritas em [Leonhardt et al. 2003] proporcionam a utilização do ambiente ALICE na criação do chatbot Elektra, que tem o objetivo de ajudar no ensino de Física, respondendo perguntas e direcionando usuários a websites contidos em seu banco de dados. Outro exemplo de uso de chatbot no Brasil é o Lassalinho proposto por [Gomes et al. 2005] criado através da interação de usuário por meio de bate-papo nos ambientes de aprendizagem.

Um dos primeiros chatbots criados foi o ELIZA [Weizenbaum 1966], o qual tinha o objetivo de emular o papel de um psicoterapeuta. Com novas técnicas de IA surgindo ao longo dos anos, elas foram sendo aplicadas à construção dos chatbots, de modo que os exemplos de sistemas podem ser categorizados, de forma geral, em três gerações: a primeira, baseada em padrões e regras gramaticais; a segunda, fundamentada nas regras de produção e nas redes neurais artificiais; e a terceira, que faz uso das linguagens de marcação AIML [Kuyven et al. 2018].

Chatbots também podem ser integrados com AVAs, como proposto por [Motta 2019], em que um chatbot foi integrado na plataforma Moodle, disponível para responder perguntas (FAQs) e tirar duvidas de estudantes. Sendo as resoluções dessas derivadas de uma base de conhecimento estabelecida pelo professor [Motta 2019].

A principal diferença deste trabalho frente aos demais está na aplicação de uma modelagem que considera recursos de MAEs. Isso permite que todo o processo de construção e adequação das ações seja claramente apresentada aos estudantes. 


\section{Modelo Proposto}

A arquitetura proposta tem como objetivo facilitar a integração de chatbots no processo de criação de ambientes educacionais. A arquitetura foi projetada de forma a permitir que o ambiente educacional possa ser integrado, independentemente da infraestrutura já existente, necessitando, somente de implementação de interfaces para comunicação com os demais métodos. Na Figura 1 é possível observar a disposição dos componentes da arquitetura.

Figura 1. Arquitetura proposta

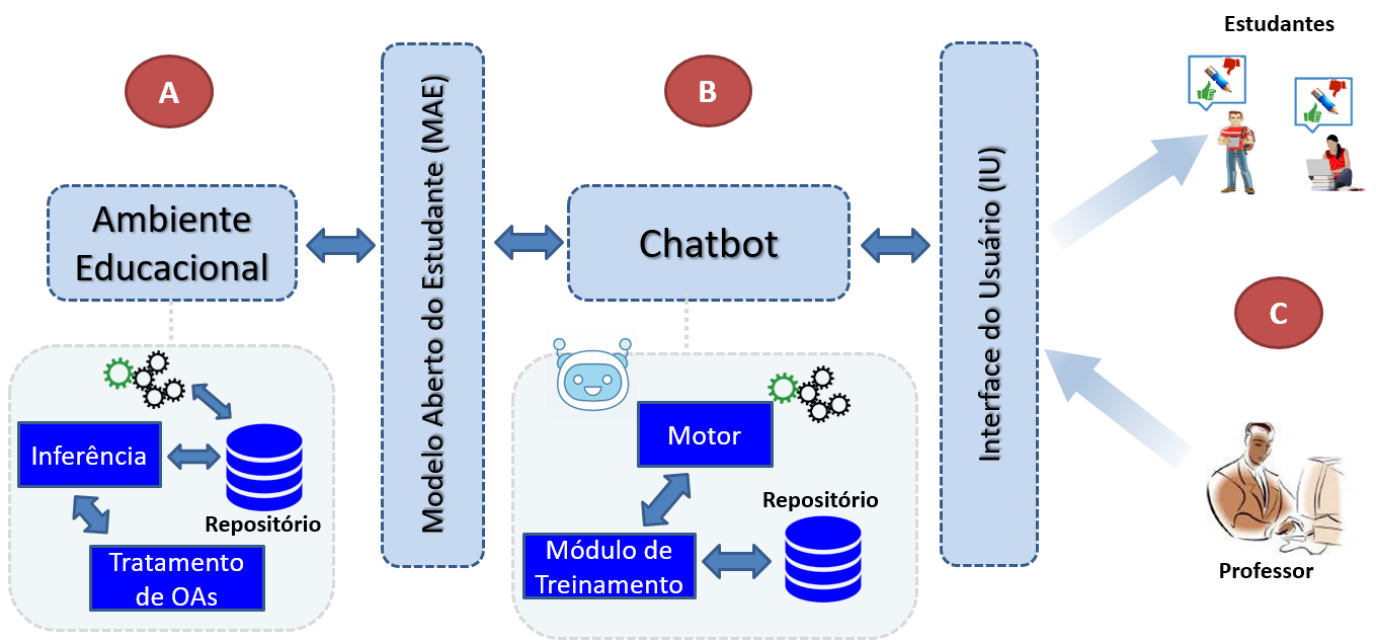

Pode-se detalhar a estrutura da arquitetura a partir de três partes: Ambiente Educacional (Figura 1-A), Chatbot (Figura 1-B) e Participantes do processo educacional (Figura 1-C). O Ambiente Educacional (A) é responsável pelo processamento sobre o nível de conhecimento dos estudantes e sobre as inferências do que será apresentado a cada estudante. Todas as interações provenientes do Chatbot (B) são encaminhadas ao Ambiente Educacional por meio do MAE, interface de comunicação entre os dois componentes (A e B); Vale ressaltar que as interações de estudantes e professores com o Chatbot acontecem através da interface do Usuário (IU), ambiente que interpreta as interações humanas e as convertem em linguagem para treinamento do Motor de Busca do Chatbot (B).

Sempre que um novo Objeto de Aprendizagem (OA) é inserido no Ambiente Educacional ele é processado e, com base nas informações sobre o comportamento do estudante, esse objeto é assim disponibilizado para sua utilização. Qualquer dúvida, questionamento ou consideração é encaminhada pelo estudante (ou professor) ao chatbot por meio da interface do Usuário (IU), que, após processada, é repassada para o MAE aplicar as alterações junto ao Ambiente Educacional. Esse processo acontece constantemente para todas as recomendações realizadas no ambiente educacional.

\section{Considerações Finais}

A área da Computação que propõe o desenvolvimento de abordagens inteligentes para auxiliar na realização de tarefas cotidianas tem ganhado atenção nos últimos anos. Esses sistemas são capazes de entender os anseios dos usuários e adaptar-se às suas capacidade e desejos. Assim, os resultados trazidos por este trabalho tendem a auxiliar as propostas de 
melhoramento no engajamento dos estudantes por meio de ferramentas que possibilitem melhor comunicação entre estudante, professor e ambiente educacional.

A arquitetura proposta integra a comunicação realizada entre ambiente educacional (por meio de um modelo de estudante aberto) com as pessoas envolvidas neste processo, como professores e estudantes. Resultados preliminares comprovaram a viabilidade da proposta e a possibilidade real de utilização da arquitetura.

Após a implementação deste trabalho alguns trabalhos futuros puderam ser vislumbrados, como: a implementação e integração da arquitetura proposta em um Ambiente Educacional Ubíquo, treinamentos para melhoria do vocabulário integrado ao Chatbot, e por fim, testes com estudantes e professores afim de verificar a eficácia do modelo.

\section{Agradecimentos}

Os autores agradecem ao CNPq, FAPEMIG e IFSULDEMINAS pelo apoio.

\section{Referências}

Brusilovsky, P. (2001). Adaptive hypermedia, user modeling and user-adapted. Interaction Journal, 11(1-2):87-110.

Durlach, P. J. and Lesgold, A. M. (2012). Adaptive technologies for training and education. Cambridge University Press.

Ferreira, H. N. M., Araújo, R. D., Dorça, F. A., and Cattelan, R. G. (2017). Open student modeling for academic performance visualization in ubiquitous learning environments. In 2017 IEEE Inter. Conf. on Systems, Man, and Cybernetics (SMC), pages 641-646.

Gomes, R. S., Barbosa, D. N. F., and Geyer, C. F. R. (2005). Lassalinho: um agente pedagógico animado em um ambiente multiagente para educação a distância. RENOTE: Revista Novas Tecnologias na Educação.

Greiff, S., Niepel, C., Scherer, R., and Martin, R. (2016). Understanding students' performance in a computer-based assessment of complex problem solving: An analysis of behavioral data from computer-generated log files. Comp. in Human Beh., 61:36-46.

Kuyven, N. L., Antunes, C. A., de Barros Vanzin, V. J., da Silva, J. L. T., Krassmann, A. L., and Tarouco, L. M. R. (2018). Chatbots na educação: uma revisão sistemática da literatura. RENOTE: Revista Novas Tecnologias na Educação, 16(1).

Leonhardt, M. D., Neisse, R., and Tarouco, L. M. R. (2003). Meara: um chatterbot temático para uso em ambiente educacional. In Simpósio Brasileiro de Informática na Educação - SBIE, volume 1, pages 81-88.

Motta, L. C. P. (2019). Chatbot para o moodle. RAAM-Revista Acadêmica Alcides Maya, $1(2): 17-27$.

Nguyen, Q., Rienties, B., Toetenel, L., Ferguson, R., and Whitelock, D. (2017). Examining the designs of computer-based assessment and its impact on student engagement, satisfaction, and pass rates. Computers in Human Behavior, 76:703-714.

Weizenbaum, J. (1966). Eliza - a computer program for the study of natural language communication between man and machine. Communications of the ACM, 9(1):36-45. 